\title{
IDENTIFICACIÓN DE VARIACIÓN GENÉTICA EN POBLACIONES DE Streptococcus agalactiae MEDIANTE AMPLIFICACIÓN ALEATORIA DE POLIMORFISMOS DE ADN
}

\section{GENETIC VARIATION IDENTIFICATION IN Streptococcus agalactiae POPULATIONS BY RANDOM AMPLIFIED OF POLYMORPHIC DNA}

Wilmer Yobany Gutiérrez Bernal, Leopoldo Antonio Arrieta Violet, Sofía Albesiano

Universidad Pedagógica y Tecnológica de Colombia (UPTC). Facultad de Ciencias. Programa de Biología. Grupo de Investigación en Genética y Biología Molecular (GEBIMOL). Tunja, Colombia. *Correspondencia: leopoldo.arrieta@uptc.edu.co, leopoldo.arrieta@uptc.edu.co, adriana.albesiano@uptc.edu.co

\section{RESUMEN}

En éste estudio se realizó la caracterización molecular mediante la técnica RAPD, usando los cebadores $10 \mathrm{~N}$ y $19 \mathrm{~N}$, en poblaciones de Streptococcus agalactiae de leche de vacas con mastitis del departamento de Boyacá-Colombia, con el fin de identificar la variabilidad genética intraespecifica. Se realizaron amplificaciones al azar, para 10 aislamientos de ubre, 12 en cantinas de recolección y tres controles negativos (Streptococcus mutans, Streptococcus faecalis, Streptococcus pneumoniae). Se encontraron perfiles más similares para las amplificaciones de las muestras tratadas con los cebadores en la leche de la ubre, que los perfiles en las muestras aisladas de cantinas. El grado de similitud entre las muestras se analizó con UPGMA, mediante el índice de Jaccard, el cual identificó mayores variaciones genéticas en aislamientos bacterianos de cantina de recolección que los de la leche de la ubre. Estas variaciones genéticas surgen de la interacción entre poblaciones de microorganismos de diferentes hatos que logran llegar a estos medios de almacenamiento, aumentando el número de polimorfismos que pueden estar relacionados con la presencia de genes de resistencia a antibióticos.

Palabras clave: RAPD, Streptococcus agalactiae, UPGMA y variación genética. 


\section{ABSTRACT}

In the present study, the molecular characterization was carried out by RAPD technique using $10 \mathrm{~N}$ and $19 \mathrm{~N}$ primers in Streptococcus agalactiae populations from milk of cows suffering mastitis in the department of Boyacá-Colombia, aiming to identify intraspecific genetic variability. Random amplifications were performed for 10 udder isolates, in 12 collection canteens and three negative controls (Streptococcus mutans, Streptococcus faecalis, Streptococcus pneumoniae). More similar profiles were found for sample amplifications treated with primers in udders milk than profiles for isolation in canteen samples. Similarity level between samples were analyzed with UPGMA, using the Jaccard index; which identified more genetic variations in S. agalactiae canteen samples than in samples collected in the udders milk. These genetic variations arise from the interaction between different microbial populations from different herds which are able to reach these storage's means, in order to increase the number of polymorphisms that related to the presence of antibiotic resistance genes.

Key words: Genetic variation, RAPD, Streptococcus agalactiae, UPGMA. 


\section{INTRODUCCIÓN}

La mastitis bovina es una enfermedad infecciosa; se diagnostica por la inflamación de las glándulas mamarias en los tejidos de la ubre, en respuesta a la presencia de uno o varios microorganismos patógenos introducidos por una lesión traumática o agentes irritantes a la hora del ordeño manual o mecánico (HERINGSTAD et al. 2000; ZHAO y LACASSE, 2008; RAMÍREZ et al. 2011). Estos microorganismos generan inflamación por un aumento en el número de células somáticas, las cuales participan en la defensa del organismo frente a la enfermedad (WOLTER et al. 2002), [4], ocasionando una disminución en la cantidad y calidad del producto lácteo, por pérdida de la funcionalidad de la ubre y por alteraciones bioquímicas de la leche (WOLTER et al. 2002;

CALDERÓN y RODRÍGUEZ, 2009; ANCASI y MARAZ, 2010; CALDERÓN et al. 2014). Existen dos tipos de mastitis: A.) La mastitis contagiosa que es causada por microorganismos como: Arcanobacterium pyogenes, Mycoplasma sp, Staphylococcus aureus, Streptococcus agalactiae; sus reservorios son la glándula mamaria y la leche de vacas infectadas y su transmisión puede ocurrir en el ordeño por medio de las manos contaminadas de los ordeñadores o por el uso de pezoneras no desinfectadas entre vacas en los ordeños mecánicos (BLOWEY, 2009; MANNING et al. 2010; PHILPOT y NICKERSON, 2012). B.) La mastitis ambiental es producida por gérmenes Gram-negativos, habitantes normales del ambiente (Enterobactersp., Escherichia coli, Klebsiella sp., Proteus sp., Pseudomonas sp. y Serratia sp.) y algunas bacterias Gram positivas (Streptococcus dysgalactiae y Streptococcus uberis) (SMITH y HOGAN, 2011). S. agalactiae se encuentra entre los principales microorganismos causantes de la mastitis, denominado en la literatura como "Estreptococo del Grupo B (EGB)", es un 
diplococo Gram positivo, capsulado, anaerobio facultativo, beta hemolítico, no esporulado, inmóvil, catalasa y oxidasa negativo; que se presenta en pares o formando cadenas cortas y se caracteriza por la presencia del antígeno polisacárido B de Lancefield (VERSALOVIC et al. 2011), la diferenciación del polisacárido capsular, la presencia de proteínas de superficie y los métodos moleculares permiten su clasificación en serotipos y genotipos (PÉREZ, 2006). La patología generada por esté microorganismo en bovinos induce a una disminución del 4 al 30\% en la producción de leche (BEDOLLA y PONCE DE LEÓN, 2008) y puede trasmitirse a neonatos y mujeres (SAVOIA et al. 2008). En Colombia, las vacas con mastitis evidencian un alto porcentaje de aislamientos de $S$. agalactiae: del total de bacterias, en el altiplano Cundiboyacense, más del 6.8\% pertenecen a este microorganismo (WOLTER, 2003; ANDRADE, 2014), para el altiplano del norte de Antioquia 34\% (RAMíREZ et al. 2011) y para la Sabana de Bogotá 35.4\%, (RODRÍGUEZ et al. 2002). La identificación definitiva de $S$. agalactiae requiere la demostración del antígeno específico de grupo, lo que presentan un alto poder de discriminación y una alta reproducibilidad, sin embargo esté método no es eficaz en distinguir entre las poblaciones de la especie (OLIVE y BEAN, 1999), presentes en el departamento; así se necesitan técnicas con marcadores moleculares que permitan identificar variabilidad entre las poblaciones de la especie; para tales fines en literatura se propone la caracterización molecular del agente infeccioso mediante diferentes técnicas, para aumentar la eficacia en el reconocimiento de las entidades bacterianas y determinar sus propiedades genotípicas (FRANCOIS y LIMANSKY, 2001; DUARTE et al. 2004; DIMITRIEV et al. 2004; CANCINO et al. 2012; RANJAN, 2012; YANG et al. 2013), entre estas se encuentra la 
técnica basada en la identificación de polimorfismos derivados de la amplificación al azar de ADN (RAPD), la cual permite la diferenciación de cepas bacterianas. La investigación tuvo como objetivo identificar la variabilidad genética intraespecífica en poblaciones de S. agalactiae en el departamento de Boyacá; para ello se buscó encontrar las relaciones de similitud, por índices de diversidad beta cualitativos (MEYER et al. 2004; Ranjan et al 2012; BARRIOS y MERCADO, 2014), entre los perfiles obtenidos por RAPD y por agrupamientos entre los perfiles de amplificación en dendogramas mediante análisis UPGMA (Unweighted Pair Group Method with Arithmetic Mean), entre poblaciones de $S$. agalactiae aisladas de la ubre de bovinos afectados por mastitis y de cantinas de recolección lechera. 


\section{MATERIALES Y MÉTODOS}

Se obtuvieron 25 aislamientos bacterianos del género Streptococcus provenientes de cuatro municipios de Boyacá (Figura 1, Tabla 1), identificados bioquímicamente según TORRES (2014); estos fueron inoculados en $2 \mathrm{ml}$ de medio de cultivo liquido HBI (Heart and Brain Infusion), e incubados a $37^{\circ} \mathrm{C}$ durante 48 horas para obtener la mayor cantidad de ADN; luego se procedió a la extracción.

Tabla 1. Localización y nomenclatura de 25 aislamientos bacterianos $C=C a n t i n a$, U=Ubre. *Muestras obtenidas del laboratorio de servicios clínicos LAC LTDA, Instituto Cancerológico de Tunja.

\begin{tabular}{|c|c|c|c|c|}
\hline Especie & Número & Procedencia & Nomenclatura & Municipio \\
\hline \multirow{6}{*}{$\begin{array}{l}\text { Streptococcus } \\
\text { agalactiae }\end{array}$} & \multirow{2}{*}{12} & \multirow{3}{*}{ Cantina } & $6(\mathrm{C} 1-\mathrm{C} 6)$ & Paipa \\
\hline & & & 6 (C7-C12) & Firavitoba \\
\hline & \multirow{4}{*}{10} & & 4 (U1-U4) & Firavitoba \\
\hline & & \multirow{3}{*}{ Ubre } & 3 (U5-U7) & Paipa \\
\hline & & & 1 (U8) & Toca \\
\hline & & & 2 (U9-U10) & Duitama \\
\hline $\begin{array}{l}\text { Streptococcus } \\
\text { mutans }\end{array}$ & 1 & * & Sm & - \\
\hline $\begin{array}{l}\text { Streptococcus } \\
\text { faecalis }\end{array}$ & 1 & * & Sf & - \\
\hline $\begin{array}{l}\text { Streptococcus } \\
\text { pneumoniae }\end{array}$ & 1 & * & Sn & - \\
\hline Total & 25 & - & - & - \\
\hline
\end{tabular}

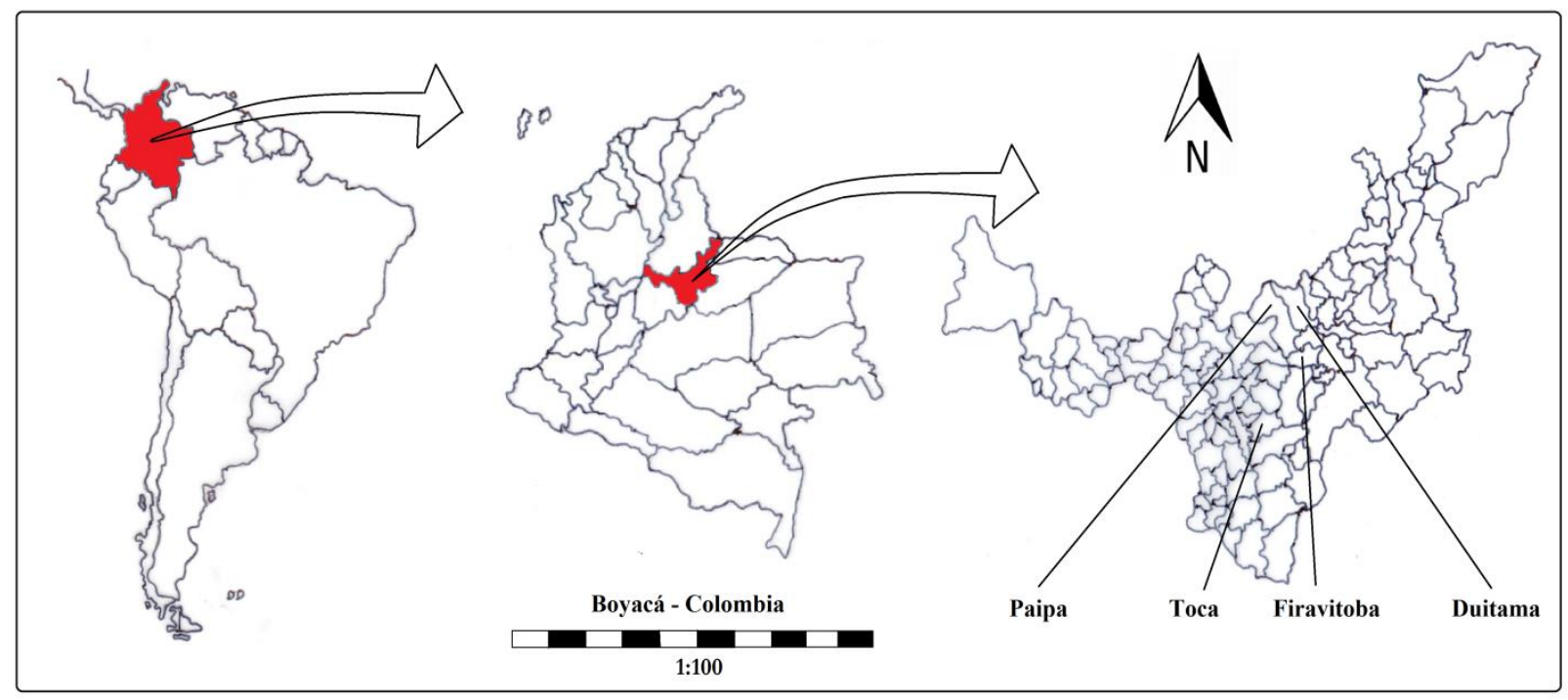

Figura 1. Mapa de ubicación de las muestras obtenidas en el departamento de Boyacá, provenientes de: la granja de Tunguavita en Paipa, y los municipios de Firavitoba, Toca y Duitama. 


\section{Extracción de ADN y amplificación RAPD}

La extracción de ADN genómico de bacterias gram positivas, se realizó con el protocolo del Kit de purificación genómico de Wizard® (Promega), de acuerdo con las instrucciones del fabricante. La caracterización genotípica por amplificación aleatoria de fragmentos polimórficos, por PCR, en termociclador Techne Prime G, se llevó a cabo en un volumen total de $25 \mu \mathrm{l}$ con: $12.5 \mu \mathrm{l}$ de GoTaq Hot Start Green Master Mix 1x [Buffer de reacción (pH 8.5), 200 M de cada uno de los dNTPs y $2 \mathrm{mM} \mathrm{MgCl} 2$ ], $4.6 \mu \mathrm{l}$ de cebadores $20 \mu \mathrm{M}$ : Cebador 10N (5'-TNGYNGGRTC-3') y $19 \mathrm{~N}$ (5'-GGTCGACYTTNGYNGGRTC-3') FRANCOIS y LIMANSKY (2001), $1.5 \mu \mathrm{l}$ de $\mathrm{MgCl}_{2}$ y $5 \mu \mathrm{l}$ de ADN extraído por muestra. La mezcla se amplificó siguiendo el protocolo de FRANCOIS y LIMANSKY (2001).

\section{Electroforesis en gel de agarosa}

El producto de la amplificación, se visualizó en un gel de agarosa al 2 \%, polimero inerte extraído de algas (MUÑOZ et al. 2012; MORENO et al. 2015)], teñido con Bromuro de Etidio, en buffer de corrido TAE (Tris HCl, Ácido acético y EDTA), a 60 voltios, durante 3 horas y 20 min. Los productos fueron visualizados en fotodocumentador modelo DIGI DOC II SYSTEM, y las bandas comparadas con un marcador de peso molecular de 2,000 pb (BioLine Reagents Ltda).

\section{Análisis fenéticos}

Para establecer la similaridad entre los perfiles obtenidos de $S$. agalactiae, se realizaron dos matrices binarias (0-1), con base en el número y tamaño molecular de los fragmentos amplificados con los cebadores $10 \mathrm{~N}$ y $19 \mathrm{~N}$. El análisis se realizó mediante el software Doc-ItLS Image Analysis 6.8 (UVA), que está diseñado para el análisis de geles en primera dimensión. Se realizaron dos dendogramas, uno con el cebador $10 \mathrm{~N}$ y el otro con $19 \mathrm{~N}$, utilizando el método de agrupamiento UPGMA, y 
con valores de similitud del índice de Jaccard, cuyos valores cercanos a 0 significan disimilitud y los valores cercanos a 1 muestran similitud.

\section{RESULTADOS}

Se encontró variación genética por diferencia en los polimorfismos presentes entre las poblaciones de $S$. agalactiae, obtenidas de aislamientos de cantinas de recolección lechera y de la leche de la ubre, identificada por la técnica RAPD con los dos cebadores (10N y 19N), donde el cebador 19N genero mayor número de bandas que el cebador $10 \mathrm{~N}$ (Tabla 2). Se obtuvieron geles con 47 perfiles de amplificación con las siguientes características: Cebador 10N-12 muestras de leche de cantinas de recolección y 10 muestras de leche de ubre: Generaron un total de 80 bandas con tamaños entre 120 y 1,500 pb para la ubre y 115 bandas entre 290 y 2,000 pb en cantina (Tabla 2, Fig. 2). Cebador 19N-12 muestras de leche de cantinas de recolección, 10 muestras de leche de ubre y los tres controles: Generaron 200 bandas con tamaños moleculares entre 290 a 2,000 pb en ubre, 181 bandas de 290 a 2,000 pb en cantina y 49 bandas en los controles, que oscilaron entre 190 y 1,600 pb (Tabla 2, Fig. 3). El perfil más común obtenido fue en ubre, el cual posee un total de 14 bandas.

Tabla 2. Polimorfismos detectados con dos cebadores al azar usados para 47 aislamientos, 44 de $S$. agalactiae y tres controles: $S$. mutans, $S$. feacalis y $S$. pneumoniae

\begin{tabular}{|llllll|}
\hline $\begin{array}{l}\text { Prime } \\
r\end{array}$ & $\begin{array}{l}\text { Proceden } \\
\text { cia }\end{array}$ & $\begin{array}{l}\text { Tamaño } \\
\text { de bandas } \\
(\mathrm{pb})\end{array}$ & $\begin{array}{l}\text { Número de } \\
\text { bandas } \\
\text { obtenidas }\end{array}$ & $\begin{array}{l}\text { Promedio de } \\
\text { bandas por } \\
\text { amplificación }\end{array}$ & $\begin{array}{l}\text { Numero de } \\
\text { bandas } \\
\text { polimórficas }\end{array}$ \\
\hline $10 \mathrm{~N}$ & Cantina & $290-2,000$ & 115 & 9.583 & 7 \\
\hline $10 \mathrm{~N}$ & Ubre & $120-1,500$ & 80 & 8 & 3 \\
\hline $19 \mathrm{~N}$ & Cantina & $290-2,000$ & 181 & 15.083 & 8 \\
\hline $19 \mathrm{~N}$ & Ubre & $290-2,000$ & 200 & 16.666 & 2 \\
\hline $19 \mathrm{~N}$ & Controles & $190-1,600$ & 49 & 16.333 & - \\
\hline
\end{tabular}

\section{Similaridad Intraespecífica}


Los valores de similitud con el índice de índice de Jaccard (IJ) en el analisis UPGMA para las amplificaciones con cebador $10 \mathrm{~N}$ se muestran en la Figura 4, se originan dos grupos. Grupo uno, de muestras de ubre grupo dos muestras de cantina. En el grupo uno las muestras que presentan valores altos de índice de Jaccard (0.97) son U1-U2 y U7-U10. Los perfiles más disimiles son U6 que presenta (IJ=0.322), U6 presenta dos de los polimorfismos con una banda de 120 pb (Fig. 2A). Y un segundo grupo que reúne los perfiles de cantina, donde C1-C2 y C3-C4 son los más similares $(\mathrm{IJ}=0.975)$, los dos primeros forman un subgrupo con $\mathrm{C} 10-\mathrm{C} 11(\mathrm{IJ}=0.578)$ y los segundos forman un subgrupo con $\mathrm{C} 5$, C6 y C7 (IJ=0.712); los perfiles C9 y C8 son muy similares entre ellos $(\mathrm{IJ}=0.961)$, pero difieren del resto del grupo de cantina $(\mathrm{IJ}=0.483)$.

Figura 2. Amplificaciones por RAPD con cebador $10 \mathrm{~N}$, de muestras de $S$. agalactiae, visualizadas en gel de agarosa al $2 \%$. Panel 2A. 10 muestras de leche tomada de ubre. Panel 2B. 12 muestras de leche tomadas de cantinas de recolección. Se anexa el documento

Figura 3. Amplificaciones por RAPD con cebador $19 \mathrm{~N}$, visualizados en gel de agarosa $2 \%$, de $S$. agalactiae. Panel 3A. Amplificaciones de muestras de la ubre. Panel 3B. 12 amplificaciones de muestras de cantinas de recolección. El panel 3C. Tres amplificaciones de los controles, Sm: $S$. mutans, Sf: $S$. faecalis y $\mathrm{Sn}$ : $S$. pneumoniae. MP: Marcador de peso molecular. Se anexa el documento

En la Figura 5. Cebador 19N, el análisis UPGMA genera tres grupos (Grupo uno: controles, grupo dos: muestras de cantina y grupo tres: muestras de ubre). El primer grupo comprende las muestras control (S. mutans, S. faecalis y S. pneumoniae) debido a su baja similitud $(\mathrm{IJ}=0.002)$, con los perfiles de amplificación de $S$. agalactiae (Fig. 3C). El segundo grupo (Cantina), con doce perfiles de $S$. agalactiae donde se agrupa C8-C9, muy similares entre ellos ( $\mathrm{IJ}=0.975)$ y con $\mathrm{C} 10, \mathrm{C} 11$ y $\mathrm{C} 12$ ( $(\mathrm{J}=0.675)$, y con $\mathrm{C} 1-\mathrm{C} 2(\mathrm{IJ}=0.908)$ como subgrupo más cercano $(\mathrm{IJ}=0.648)$; las amplificaciones del subgrupo $\mathrm{C} 3, \mathrm{C} 4$ y C5 son más similares con C6 y C7 (IJ= 
0.475), que con el resto (Fig. 3B y Fig. 5). El grupo tres (Ubre), con perfiles muy similares para U1 a U3 (IJ=0.973) con U4 como más similar ( $I J=0.895)$, unido a un

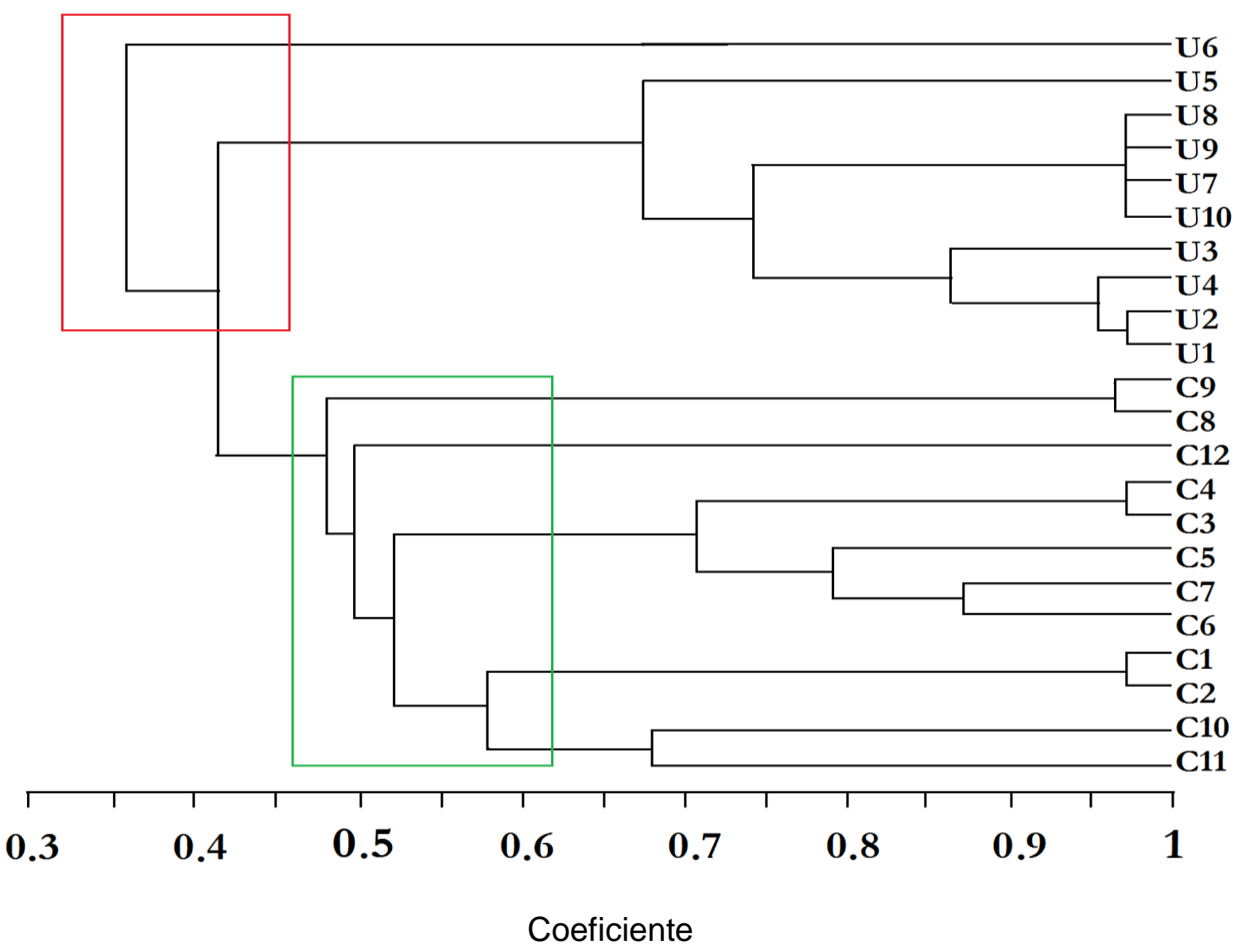

Figura 4. Dendograma para RAPD de 22 perfiles de amplificación de S.agalactiae. 12 provenientes de cantinas de recolección lechera $(\mathrm{C} 1$ a $\mathrm{C} 12)$ y 10 provenientes de leche de ubre (U1 a U10) amplificadas con cebador $10 \mathrm{~N}$, con valores de similitud mediante el índice de Jaccard.

subgrupo de U6 a U10 (IJ=0.725), siendo U7, U9 y U10 las más similares dentro de ese subgrupo ( $\mid \mathrm{J}=0.975)$, mientras que U5 se presenta separado de los dos subgrupos anteriores tanto en el gel como en el dendograma ( $\mathrm{IJ}=0.625)$, (Fig. 3A y Fig. 5).

\section{Similaridad Interespecífica}

A diferencia de los controles todos los perfiles de las muestras no presentan amplificación de fragmentos menores de 250 pb (Tabla 2) excepto una banda 
polimórfica en el perfil U6 que lo coloca como el perfil más diferente de las amplificaciones con el cebador 19N, en el análisis UPGMA, esta característica en la cual difieren los perfiles de amplificación de las pruebas realizadas con las cuatro especies del mismo género, debe estar incluido en la descripción de la caracterización genotípica. En relación con los resultados interespecíficos, las amplificaciones con el cebador $19 \mathrm{~N}$ para las muestras control, no muestran similitud con ninguno de los perfiles de $S$. agalactiae demostrando la utilidad de la técnica en la diferenciación genotípica entre organismos. El perfil de la especie $S$. mutans es más similar al generado por $S$. pneumoniae $(\mathrm{IJ}=0.902)$ que a $\mathrm{S}$. faecalis $(\mathrm{IJ}=0.758)$.

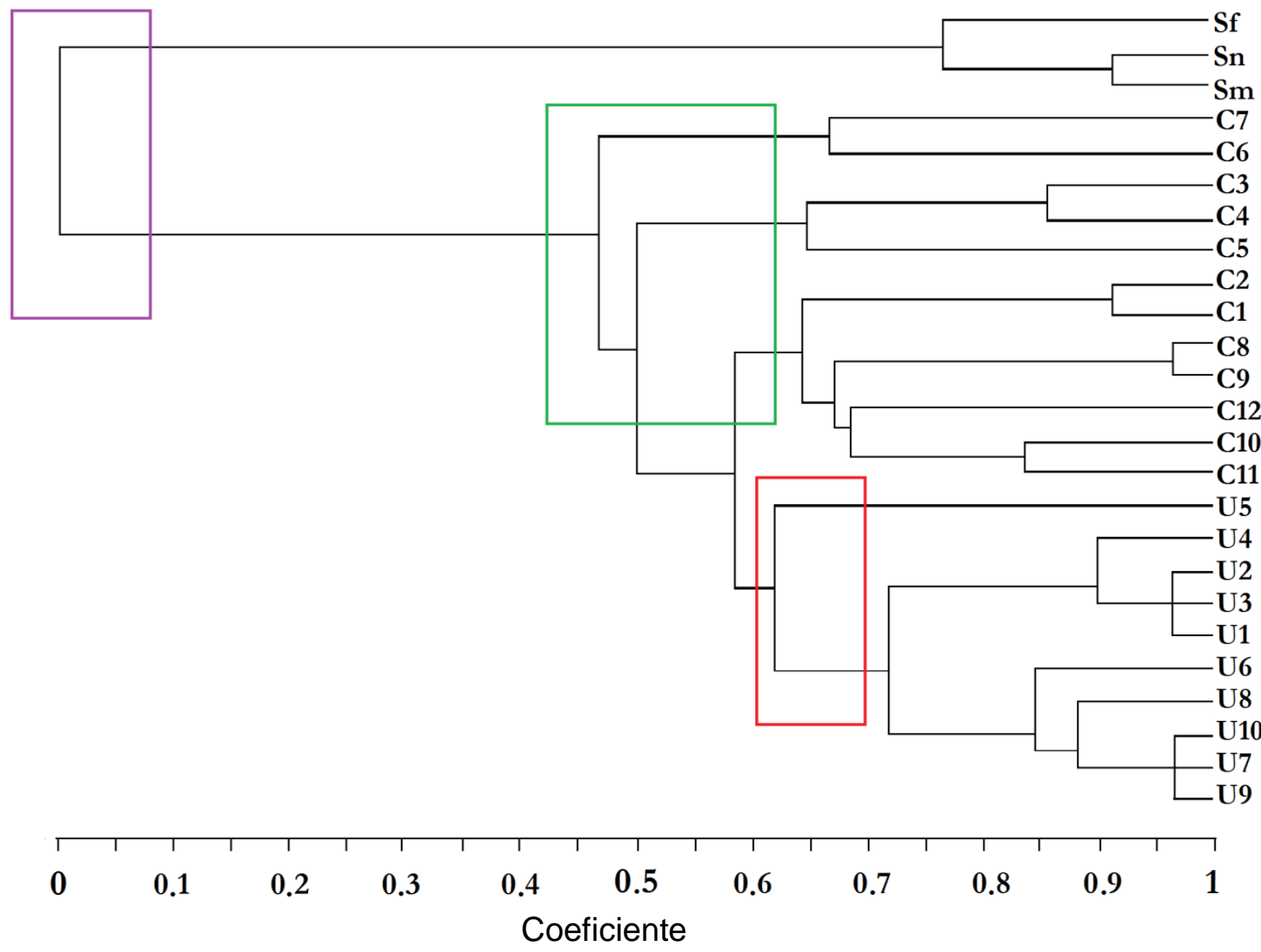

Figura 5. Dendograma para RAPD de 22 muestras de $S$. agalactiae, 12 provenientes de cantina (C1 a C12), 10 muestras de ubre (U1 a U10) y 3 muestras control Streptococcus mutans (Sm), Streptococcus faecalis (Sf) y Streptococcus pneumoniae (Sn), amplificadas con cebador $19 \mathrm{~N}$ con valores de similitud mediante el índice Jaccard. 


\section{DISCUSIÓN}

Las concentraciones de ADN obtenidas en las extracciones originaron perfiles con bandas brillantes en los geles de agarosa al $2 \%$, la utilización de buffer TAE presentó bandas más nítidas que el TBE, lo que indica que el ácido acético es más útil que el ácido bórico en la visualización de fragmentos mayores a 300 pb, estas condiciones estandarizadas de extracción y amplificación generaron perfiles más claros que los obtenidos por (FRANCOIS y LIMANSKY, 2001) y (LIMANSKY et al. 1998), de donde se escogieron los cebadores. La técnica RAPD, puede detectar un número importante de polimorfismos en el material genético de $S$. agalactiae, tanto el cebador $10 \mathrm{~N}$ como el $19 \mathrm{~N}$ separaron en dos grupos las muestras de cantina y las de ubre, lo que indica la disimilitud entre estas dos muestras (Figs. 4 y 5 ). La amplificación con el cebador 10N mostró un número menor de bandas en comparación a las obtenidas con el cebador 19N; la prueba confirma la utilidad de la técnica utilizada, como un procedimiento de laboratorio sencillo y práctico en el análisis de variabilidad genotípica de $S$. agalactiae, el cual permite la caracterización molecular con los patrones más comunes en los RAPD de las poblaciones aisladas del departamento de Boyacá, con los dos marcadores moleculares utilizados. Las pruebas con el cebador $19 \mathrm{~N}$ en poblaciones tomadas de las ubres permitieron ver un patrón de amplificación más homogéneo y propio de la especie, el cual puede usarse como técnica de identificación del patógeno. Los resultados de la técnica difieren a la hora de aplicar varios cebadores al azar, el número de bandas polimórficas varía y los agrupamientos en los análisis no son iguales, pero puede indicar que las poblaciones tienen un origen cercano, así los perfiles originados de poblaciones del mismo hato tendrán perfiles mucho más similares entre ellas, que con otros hatos. 
De acuerdo con los resultados de los dendogramas se observa que las muestras de leche de ubre son similares originándose solo tres perfiles diferentes que corresponderían a variaciones intraespecíficas. La mezcla de poblaciones de diferentes ubres y hatos en la cantina genera siete perfiles diferentes, lo que se puede interpretar con recombinación genética.

Los perfiles de las amplificaciones de las muestras indican que existe más variabilidad genotípica intraespecífica en poblaciones de $S$. agalactiae que se aislaron de cantinas de recolección, que las poblaciones de la ubre, la cual se determina por la relación obtenida en valores de similitud entre los perfiles obtenidos por RAPD. Para analizar el grado de similitud entre los perfiles necesitamos tener en cuenta las fuentes u orígenes de la variabilidad genotípica obtenida: según CHOPRA (2005), los polimorfismos de fragmentos amplificados se deben a: 1.) sustitución de bases o eliminación de los sitios de cebado, 2.) inserciones que hacen que los sitios de cebado demasiado distantes para apoyar amplificación o 3.) Inserciones o deleciones que cambian el tamaño del fragmento amplificado; y si indagamos más a fondo encontraremos fundamentos de genética bacteriana y estudios (LUAN, 2006; MIRZA et al. 2010), que describen que la principal fuente de recombinación se realiza por transferencia horizontal de genes (HGT), eventos que juegan un papel importante en la adaptación de poblaciones bacterianas a nuevos ambientes, que por selección de alelos originados por mutación, les confieren alguna ventaja (WIEZER y MERKL, 2005). Esté flujo genético horizontal entre bacterias de diferentes poblaciones puede ocurrir por conjugación (ZATYKAA y THOMASA, 1998), transformación (COHENS et al 1972) o transducción (THOMPSON 1994), procesos básicos de transferencia horizontal de genes y plásmidos entre bacterias. Son varios elementos genéticos identificados los que 
participan en dicha transferencia, de los cuales los más conocidos son los plásmidos autotransferibles o movilizables (AMABILE y CHICUREL,1992), transposones conjugativos y no conjugativos (SALYERS et al. 1995) y más recientemente, los integrones y casetes genéticos de resistencia (STOKES y HALL, 1989). Aunque los integrones no pueden realizar transposición, se asocian frecuentemente a secuencias de inserción o bien, a transposones y plásmidos conjugativos que les sirven como vehículos para su transmisión inter e intra especie. Estos elementos, han sido detectados en bacterias aisladas de ambientes acuáticos (ROSSER y YOUNG,1999) y de animales domésticos (GOLDSTEIN et al. 2001), lo cual refleja su amplia diseminación en la naturaleza. Como también, se ha demostrado la presencia de integrones en microorganismos ancestrales de diferentes géneros, corroborando que estas estructuras son antiguas y que han evolucionado conjuntamente con el genoma bacteriano (SABATÉ y PRATS, 2002; PÉREZ et al. 2013). (WHITE et al. 2001), afirman que la diseminación de genes de resistencia aumenta considerablemente cuando ellos forman parte de casetes genéticos móviles, lo cual los habilita para su transferencia horizontal. Por lo tanto, es claro que los integrones funcionan como sistemas de captación de genes que confieren ventajas selectivas para las bacterias, lo que les permite una rápida adaptación a los cambios ambientales y es bien conocido el rol de plásmidos y transpones en la multirresistencia de las bacterias a los antibióticos y en la diseminación natural de los determinantes de resistencia (SALYERS y AMABILE, 1997), como también se observa que los genes que confieren resistencia a ciertos antibióticos y que son idénticos (DOUCET et al. 1992; COURVALIN, 1994), tanto en bacterias Gram positivas como en las Gram negativas (GONZÁLEZ et al. 2004), como es el caso del $S$ agalactiae. El conocimiento de éste fenómeno de transformación bacteriana debe 
ser tenido en cuenta a la hora de implementar programas de uso adecuado de antimicrobianos y de normas básicas de control de infecciones (BIBEK et al. 2012). Por lo que no se debe aplicar el mismo antibiótico a las diferentes cepas, el control de estas cepas sin duda mejorará la calidad de los métodos de producción y comercialización de leche en los hatos de Boyacá.

En nuestro trabajo esta variación expresada en el aumento del número de polimorfismos se da por intercambio de fragmentos entre poblaciones que interaccionan en los recipientes de recolección lechera; este análisis es importante, tendiente a identificar focos de variabilidad y a realizar un tratamiento más acertado de las patologías generadas por $S$. agalactiae, ya que por medio de la identificación de las cepas y la identificación de variabilidad genética, se puede dimensionar la incidencia de este patógeno y puede servir de referencia para estudios que pretendan comprender tanto los nichos de poblaciones muy variables, como proveer herramientas que faciliten el control en las cadenas de transmisión, generando un impacto positivo en la industria lechera. Como sabemos, los estudios de genética molecular de cepas de campo de $S$. agalactiae son de vital importancia, por lo cual se propone realizar pruebas con un mayor número de cebadores que identifiquen los patrones de polimorfismos propios para las especies del género Streptococcus causantes de patologías, para ampliar la genotipificación de las poblaciones presentes en el departamento y del país, con el fin de identificar cambios genotípicos drásticos e implementar prácticas agrícolas más eficientes. 


\section{CONCLUSIONES}

* La técnica RAPD (usando los cebadores $10 \mathrm{~N}$ y 19N), identificó la variabilidad genética interespecífica en Streptoccocus e intraespecífica en S. agalactiae. Esta variabilidad indica malas prácticas pecuarias, generadas por la recombinación en la propagación alélica entre diferentes poblaciones de las zonas muestreadas. El cebador 19N ubicó más sitios de unión al genoma de S. agalactiae que el cebador $10 \mathrm{~N}$.

* Tanto el cebador $10 \mathrm{~N}$ como el $19 \mathrm{~N}$ separaron en dos grupos las muestras de cantina y las de ubre, lo que indica la disimilitud o variación genética entre las poblaciones aisladas de estos dos tipos de muestra.

* La variabilidad genética de las muestras de leche colectadas de la ubre fue menor a las muestras de leche tomadas de las cantinas de recolección, lo que indica que la recolección lechera por cantinas aumenta la probabilidad de flujo de genes y plásmidos por transferencia horizontal en poblaciones de $S$. agalactiae, incrementando la variabilidad genotípica y la propagación de alelos de resistencia a antibióticos.

* Es necesario que los procedimientos realizados por la industria lechera disminuyan la propagación de $S$. agalactiae, ya que está directamente relacionado con las perdidas en la producción por mastitis.

\section{* AGRADECIMIENTOS}

- A la Dirección de Investigaciones (DIN) de la Universidad Pedagógica y Tecnológica de Colombia, por facilitar los recursos e instalaciones para llevar a cabo la investigación, a María Inés Torres y Alix Dallos por la caracterización bioquímica de las cepas y al Grupo de Investigación en Medicina Veterinaria y Zootecnia (GIDIMEVETZ). 


\section{BiBLIOGRAFía}

Amabile C, Chicurel M (1992). Bacterial plasmids and gene flux. Cell 70(2): 189-199.

* Ancasi EG, Maraz FA (2014). Biodiversidad y estudio biotecnológico de levaduras en quesos de cabra procedentes de valles y de la Quebrada de Humahuaca-Jujuy-Argentina. Bistua 12(2): 24-34.

* Andrade R, Caro Z, Dallos A (2014). Prevalencia de mastitis subclínica bovina y su etiología infecciosa en fincas lecheras del altiplano boyacense (Colombia). FCV-LUZ 24(4): 305-310.

* Barrios E, Mercado J (2014). Plantas útiles del corregimiento Santa Inés y la vereda San Felipe (San Marcos, Sucre, Colombia). Ciencia en Desarrollo 5(2): $131-144$.

* Bedolla C, Ponce de León MER (2008). Pérdidas económicas ocasionadas por la mastitis bovina en la industria lechera". REDVET 9(4): 1695-1705.

* Bibek R, Mani B, Susweta DM, Natesan K, Rajeswari S, Dhanikachalam V, Apala B, Sukhadeo BB, Krishnamshetty P, Habibar R (2012). Molecular characterization of Streptococcus agalactiae and S. uberis isolates from bovine milk. Trop Anim Health Prod 44(8): 1981-1998.

* Blowey R (2009). Mastitis. En Control de la mastitis en granjas de vacunos de leche. Guía práctica e ilustrada. P. Edmonson (ed.). Acribia Zaragoza. España, pp 208-209

* Calderón A, Rodríguez VC (2009). Prevalencia de mastitis bovina y su etiología infecciosa en sistemas especializados en producción de leche en el altiplano Cundiboyacense (Colombia). Rev Colomb Cienc Pec 21(4): 582-589. 
* Calderón A, Arteaga M, Rodríguez V, Arrieta G, Vergara O (2014). Efecto de la mastitis subclínica sobre la calidad físico-química de la leche bovina. FCVLUZ 24(55): 408-413.

* Cancino G, Barbosa D, Carvajal C (2012). Diversidad genética de especies silvestres y cultivadas de Rubus L. de los municipios de Pamplona y Chitagá, región Nororiental de Colombia. Bistua 10(1): 80-89.

* Chopra VL (2005). Mutagenesis: investigating the process and processing the outcome for crop improvement. Curr Sci 89(2): 353-359.

* Cohens N, Chang A, Hsu L (1972). Nonchromosomal antibiotic resistance in bacteria: genetic transformation of Escherichia coli by R-factor DNA. Proc Natl Acad Sci 69(8): 2110-2114.

* Courvalin P (1994). Transfer of antibiotic resistance genes between grampositive and gram-negative bacteria. Antimicrob Agents Chemother 38(7): $1447-1451$.

* Dmitriev A, Suvorov A, Shen A, Yang Y (2004). Clinical diagnosis of group B streptococci by scpB gene based PCR. Indian J Med Res 119(Suppl): 233236.

* Doucet F, Trieu P, Andremont A, Courvalin P (1992). Conjugal transfer of plasmid DNA from Enterococcus faecalis to Escherichia coli in digestive tracts of gnotobiotic mice. Antimicrob Agents Chemother 36(2): 502-504.

* Duarte R, Miranda O, B. Bellei, M. Aparecida, V. Britond (2004). Phenotypic and molecular characteristics of Streptococcus agalactiae isolates recovered from milk of dairy cows in Brazil. J Clin Microbiol 42(9): 4214-4222. 
* François S, Limansky A, Toresani I, Ebner G, Viale A, Sutich E (2001). Caracterización de Streptococus causante de mastitis bovina en Argentina mediante métodos fenotípicos y genotípicos. Rev Mex Vet 32(4): 305-309.

* Goldstein C, Lee M, Sánchez S, Hudson C, Phillips B, Register B, Grady M, Liebert C, Summers AO, White DG, Maurer JJ (2001). Incidence of class 1 and 2 integrases in clinical and commensal bacteria from livestock, companion animals, and exotics. Antimicrob Agents Chemother 45(3): 723-726.

* González G, Mella S, Zemelman R (2004). Integrones y cassettes genéticos de resistencia: estructura y rol frente a los antibacterianos. Rev Méd Chile 132(5): 619-626.

* Heringstad B, Klemetsda G, Ruane J (2000). Selection for mastitis resistance in dairy cattle: A review with focus on the situation in the nordic countries. Livest Prod Sci 64(2): 95--106.

* Limansky A, Sutich EG, GuardatI MC, Toresani IE, Viale AM (1998). Genomic diversity among Streptococcus agalactiae isolates detected by a degenerate oligonucleotide-primed amplification assay. J Infect Dis 177(5): 1308-1313.

* Luan S (2006). Molecular epidemiology of Streptococcus agalactiae: mobile elements as genetic markers. Umea University Medical Dissertations. http://umu.diva-portal.org/smash/get/diva2:144850/FULLTEXT01.pdf

* Manning S, Springman A, Million A, Milton N, McNamara S (2010). Association of group B Streptococcus colonization and bovine exposure: a prospective cross-sectional cohort study. PLoS One 5(1): e8795.

Meyer AS, García AAF, Souza AP, Souza CL (2004). Comparison of similarity coefficients used for cluster analysis with dominant markers in maize (Zea mays). Genet Mol Biol 27(1): 83-91. 
* Mirza H, Anwar M, Bokhari S (2010). In silico identification of potential horizontal gene transfer events between Archaea and pathogenic bacteria. $J$ Bioinform Seq Anal 2(3): 36-41.

* Moreno L, Muñoz E, Casanova H (2015). Flocculation with chitosan of microalgae native of the Colombian plateau. Ciencia en Desarrollo 6(1): 25-31.

* Muñoz E, Rivas B, Sánchez J (2012). Natural polymer grafted with synthetic monomer by microwave for water treatment a review. Ciencia en Desarrollo 4(1): 219-240.

* Olive D, Bean P (1999). Principles and applications of methods for DNA-based typing of microbial organisms. J Clin Microbio/ 37(6): 1661-1669.

* Pérez C (2006). Revisión sobre mastitis bovina causada por Streptococus agalatiae. Universidad Michoacana de San Nicolás de Hidalgo, Facultad de Medicina Veterinaria y Zootecnia. Michoacán, México, pp 44.

* Pérez M, Ruiz D, Schneider M, Autino J, Romanelli G (2013). La química verde como fuente de nuevos compuestos para el control de plagas agrícolas. Ciencia en Desarrollo 4(2): 83-91.

* Philpot N, Nickerson S (2012). Agentes patógenos. En: La lucha contra la mastitis en el ganado. Naperville, USA y Oelde, Germany, pp 32.

* Prieto, E. M., Alquisira, J. P., \& Alonso, C. R. (2013). Microwave and Ultrasound Activation Effect on Cationization of Corn and Potato Starches. Ciencia en Desarrollo, 4(1), 151-174.

* Ramírez N, Arroyave O, Cerón, Jaramillo M, Palacio G (2011). Factores asociados a mastitis en vacas de la microcuenca lechera del altiplano norte de Antioquia, Colombia. Rev Med Vet 22(11): 31-42. 
* Ranjan B, Bhuvana M, Mitra SD, Krithiga N, Shome R, Velu D, Banerjee A, Barbuddhe S, Prabhudas K, Rahman H (2012). Molecular characterization of Streptococcus agalactiae and Streptococcus uberis isolates from bovine milk. Trop Anim Health Prod 44(8): 1981-1992.

* Rodríguez G, Contreras D, Ordóñez M (2002). Caracterización de la mastitis bovina en el valle de Ubaté. Rev Med Vet 2(4): 57-66.

* Rosser S, Young H (1999). Identification and characterization of class 1 integrons in bacteria from an aquatic environment. $J$ Antimicrob Chemother 44(1): 11-18.

* Sabaté M, Prats G (2002). Estructura y función de los integrones. Enferm Infecc Microbiol Clin 20(7): 341-345.

* Salyers A, Shoemaker N, LI L, Stevens A (1995). Conjugative transposons: an unusual and diverse set of integrated gene transfer elements. Microbiol Rev 59(4): 575-590.

* Salyers AA, Amabile CF (1997). Why are antibiotic resistance genes so resistant to elimination? Antimicrob Agents Chemother 41(11): 2321-2325.

* Savoia D, Gottimer C, Crocilla C, Zucca M (2008). Streptococcus agalactiae in pregnant women: phenotypic and genotypic characters. J Infect 56(2): 120125.

* Smith K, Hogan J (2011). Environmental mastitis. Vet. Clin North Am Food Anim Pract 9: 489-498.

* Stokes H, Hall R (1989). A novel family of potentially mobile DNA elements encoding site specific gene integration functions: integrons. Mol Microbiol 3(12): 1669-1683.

* Thompson B (1994). Bacterial antibiotic resistance and evolution. Reason and 
Revelation 14: 61-63.

* Torres M (2014). Caracterización fenotípica y genotípica de cepas de Streptococcus agalactiae aisladas de casos de mastitis bovina en hatos del departamento de Boyacá-Colombia. Tesis de Maestría. Universidad Pedagógica y Tecnológica de Colombia.

* Versalovic J, Carroll K, Funke G (2011). Streptococcus. Manual of Clinical Microbiology 20: 331-349.

* White P, Mciver C, Rawlinson W (2001). Integrons and gene cassettes in the Enterobacteriaceae. Antimicrob Agents Chemother 45(9): 2658-2661.

* Wiezer A, Merkl R (2005). A comparative categorization of gene flux in diverse microbial species. Genomics 86(4): 462-475.

* Wolter W, Castañeda V, Kloppert B, Zschoeck M (2002). La mastitis bovina. http://geb.unigiessende/geb/volltexte/2002/912/pdf/p020003.pdf.

* Yang Y, Liu Y, Ding Y, Yi L, Ma Z, Fan H, Lu C (2013). Molecular characterization of Streptococcus agalactiae isolated from bovine mastitis in eastern China. PLoS One 8(7): 1-8.

* Zatykaa M, Thomasa C (1998). Control of genes for conjugative transfer of plasmids and other mobile elements. FEMS Microbiol Rev 21(4): 291-319.

* Zhao X, Lacasse P (2008). Mammary tissue damage during bovine mastitis: causes and control. J Anim Sci 86 (13 Suppl): 57-65. 


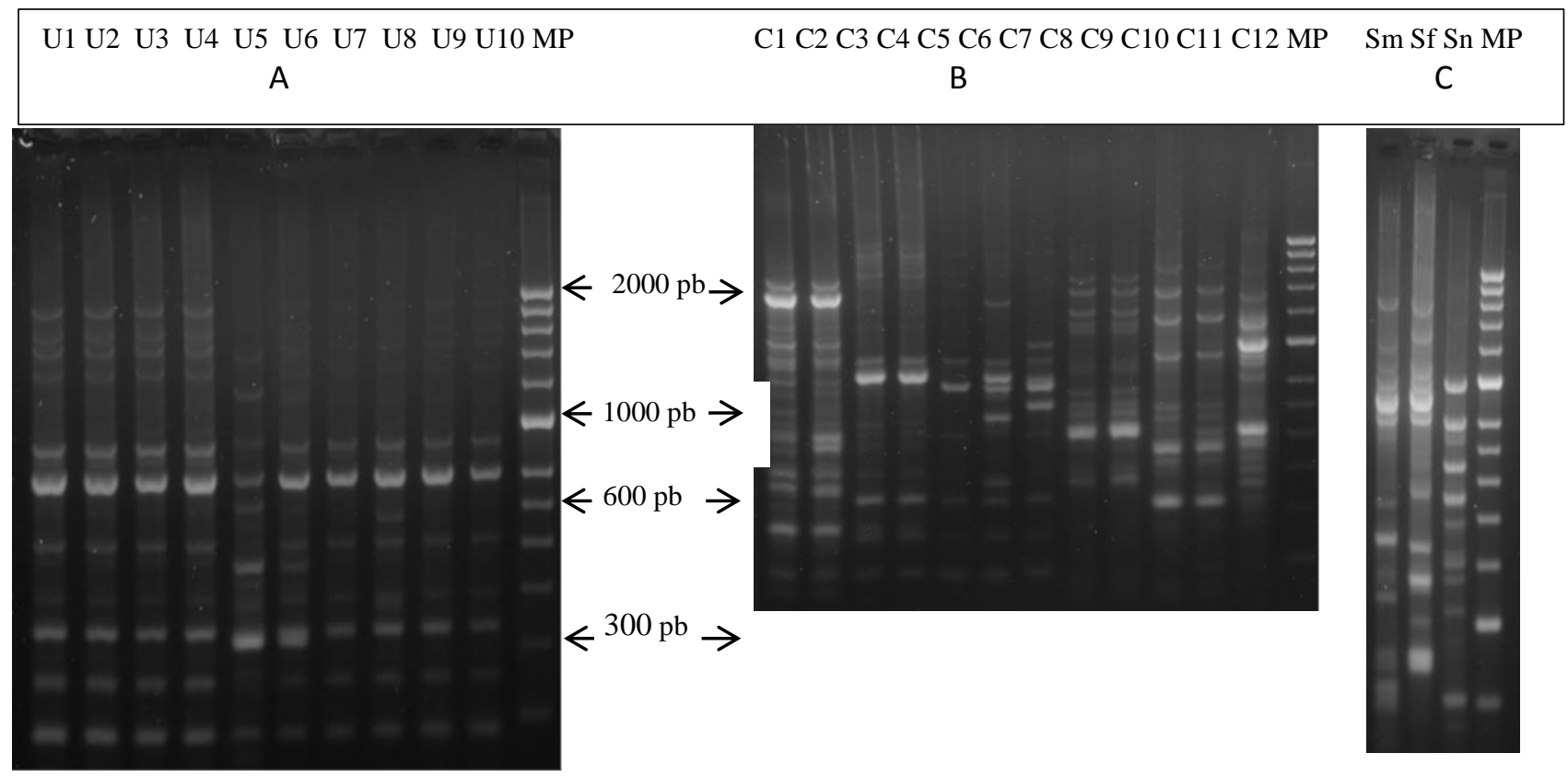

Figura 2. Amplificaciones por RAPD con el cebador $19 \mathrm{~N}$, visualizados en gel de agarosa, de $S$. agalactiae. Panel 2A. 10 amplificaciones de muestras de leche tomadas de la ubre. Panel 2B. 12 amplificaciones de muestras de leche tomadas de cantinas de recolección. El panel 2C. Tres amplificaciones de los controles, Sm: Streptococcus mutans, Sf: Streptococcus fecalis y Sn: Streptococcus neumonía. MP: Marcador de Peso Molecular.

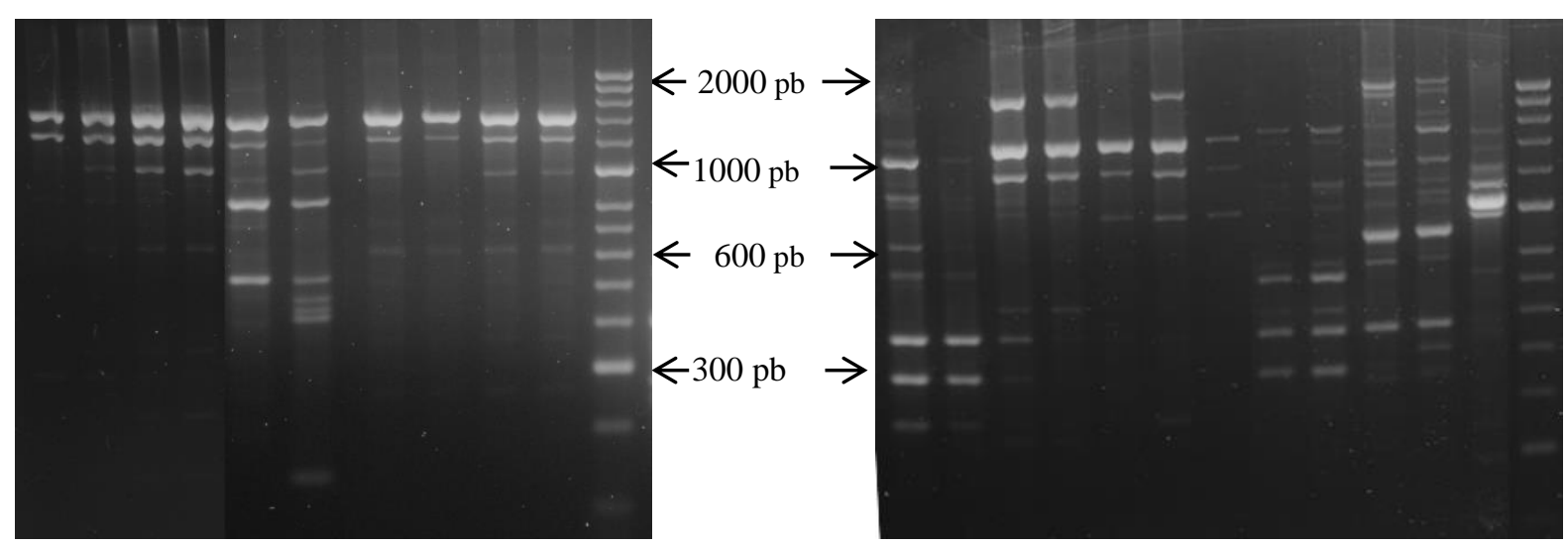

Figura 3. Amplificaciones por RAPD con cebador $10 \mathrm{~N}$, de muestras de $S$. agalactiae, visualizadas en gel de agarosa. Panel 3A. 10 muestras de leche tomada de ubre. Panel 3B. 12 muestras de leche tomadas de cantinas de recolección. 